\title{
Differentiating Rheumatoid Arthritis Related Musculoskeletal Pain in Patients With Concurrent Sickle Cell Disease: A Case Series and Literature Review
}

\author{
John Muthu ${ }^{\mathrm{a}}$, Mir Alib, c, Tracian James-Goulbourne ${ }^{\mathrm{a}}$
}

\begin{abstract}
Rheumatoid arthritis (RA) can occur concurrently in patients with sickle cell disease (SCD). Both conditions can have similar musculoskeletal symptoms during painful exacerbations. SCD tends to be frequently labelled as the causative factor of painful events by both patients and providers alike. It is important to distinguish between these two conditions causing painful exacerbations to guide treatment plans. Our review of literature showed that the concurrent presentation of RA and SCD is not well described. We present a case series of eight patients with concurrent RA and SCD. We conducted a retrospective review of their charts to make observations about differences in musculoskeletal symptoms, biomarkers and radiological changes. Generally, RA affects smaller joints compared to SCD which affects larger joints. Radiological differences include bone infarcts and avascular necrosis in SCD versus erosive arthritis in RA. In terms of biomarkers, we are better guided using C-reactive protein (CRP) in conjunction with lactate dehydrogenase (LDH). Our case series demonstrates certain indicators which help make a clearer distinction between the two usually painful conditions.
\end{abstract}

Keywords: Sickle cell disease; Rheumatoid arthritis; Musculoskeletal pain

\section{Introduction}

Sickle cell disease (SCD) is a genetic disorder characterized by presence of abnormal hemoglobin $\mathrm{S}$, which leads to sickling of RBCs when deoxygenated. Repeated vaso-occlusive pain episodes are the hallmark of SCD [1]. It involves multiple organs with musculoskeletal system being the most common site. It is estimated that SCD affects 70,000 - 100,000 Americans [2]. $\mathrm{SCD}$ is a major health concern with high rates of emergency de-

Manuscript accepted for publication June 30, 2016

aSickle Cell Division, Department of Medicine, New York City Health and Hospitals/Kings County, Brooklyn, NY 11203, USA

' Interfaith Medical Center, Brooklyn, NY 11213, USA

${ }^{\mathrm{c} C o r r e s p o n d i n g ~ A u t h o r: ~ M i r ~ A l i, ~ I n t e r f a i t h ~ M e d i c a l ~ C e n t e r, ~ B r o o k l y n, ~ N Y ~}$ 11213, USA. Email: drmirali@yahoo.com

doi: http://dx.doi.org/10.14740/jmc2602e partment (ED) visits and hospitalizations in these patients [3]. Pain is the most common reason for which patients seek medical help [4]. There could be other systemic musculoskeletal disorders which can present with similar symptoms and those diagnoses can be missed. Rheumatoid arthritis (RA) is a symmetric, inflammatory, peripheral polyarthritis of auto-immune etiology. The disease onset of RA is usually insidious with predominant symptoms being pain, stiffness and swelling of multiple joints [5]. In 2005, an estimated 1.5 million (0.6\%) of US adults had RA [6]. In our review of literature, we found only a few case reports describing these overlapping conditions. Based on review of cases with these overlapping conditions, there is often a delay in diagnosis of RA. This is mainly due to overlapping musculoskeletal symptoms which are frequently incorrectly attributed to patient's SCD. Early recognition and treatment of RA in these patients with disease modifying anti-rheumatic drugs (DMARDs) is important to achieve disease control and to prevent joint destruction and disability. Our case series demonstrates that a systematic evaluation of clinical symptoms, biomarkers and radiologic findings can help differentiate the two conditions.

\section{Case Reports}

We present eight patients who have overlapping SCD and RA. All patients had a diagnosis of SCD since childhood. RA symptoms started later in adult life. All patients diagnosed with RA met 2010 ACR/EULAR criteria which included number and site of joints, serological abnormalities (rheumatoid factor (RF) and anti-cyclic citrullinated peptide (anti-CCP)) and elevated acute phase reactants (erythrocyte sedimentation rate (ESR) and C-reactive protein (CRP)) [7]. Table 1 shows the baseline characteristics of these patients at time of diagnosis. We divided our patients into two groups based on the severity of joint involvement. Cases 1-4 had more severe joint destruction as compared to cases 5-8.

\section{Case 1}

This patient has florid RA. He had rare presentations for sickle cell related events up until diagnosis of RA after which he started to have multiple ED visits and hospitalizations for painful events. His pain during these exacerbations was localized to the knees, elbows and left shoulder. There was a diagnostic 
Table 1. Baseline Characteristics of Patients at the Time of Diagnosis With RA

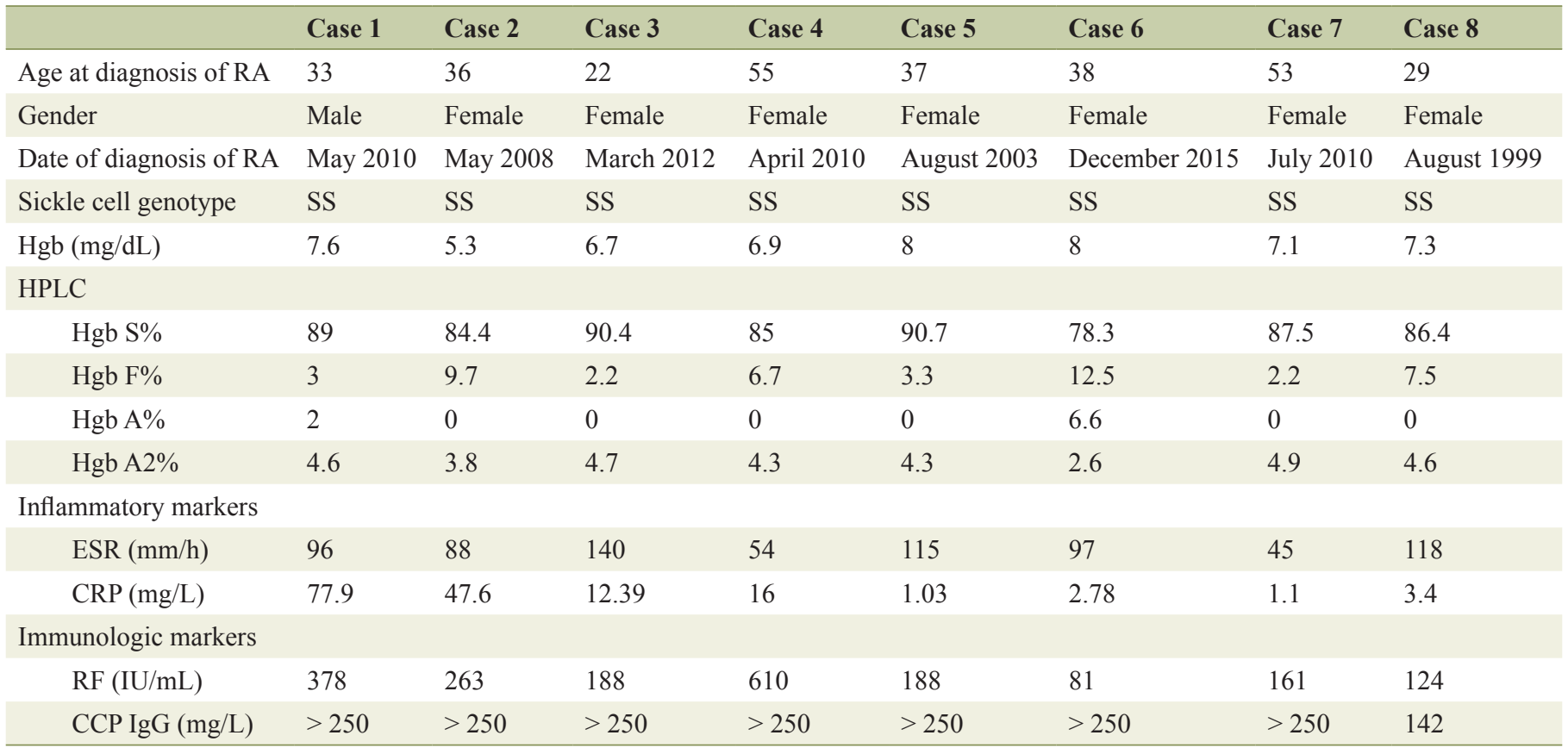

Hgb: hemoglobin; HPLC: high performance liquid chromatography; Hgb S: sickle cell hemoglobin; Hgb F: fetal hemoglobin; Hgb A\&A2: adult hemoglobins; ESR: erythrocyte sedimentation rate; CRP: C-reactive protein; RF: rheumatoid factor antibody; CCP IgG: cyclic citrullinated peptide antibody.

dilemma during each of these episodes as to whether his pain was from sickle cell crises or RA exacerbations. Over the last 6 years, he developed significant joint deformities involving the knees and elbows, subsequently becoming wheel chair bound. $\mathrm{X}$-ray of knees shows sclerotic changes from bone infarcts in the diaphysis and metaphysis due to SCD and erosive articular changes from RA (Figs. 1 and 2). The elbow X-ray showed only erosive articular change (Fig. 3). On the other hand, his left shoulder X-ray showed avascular necrosis (AVN) of the humeral head with preserved joint space (Fig. 4). He was tried on several anti-rheumatic medications including methotrexate, adalimumab and certolizumab but had an inadequate response to these. He is currently on low dose prednisone, tofacitinib and leflunomide.

\section{Case 2}

This patient has an added history of recurrent stroke, Moyamoya syndrome and residual right-sided weakness. She did

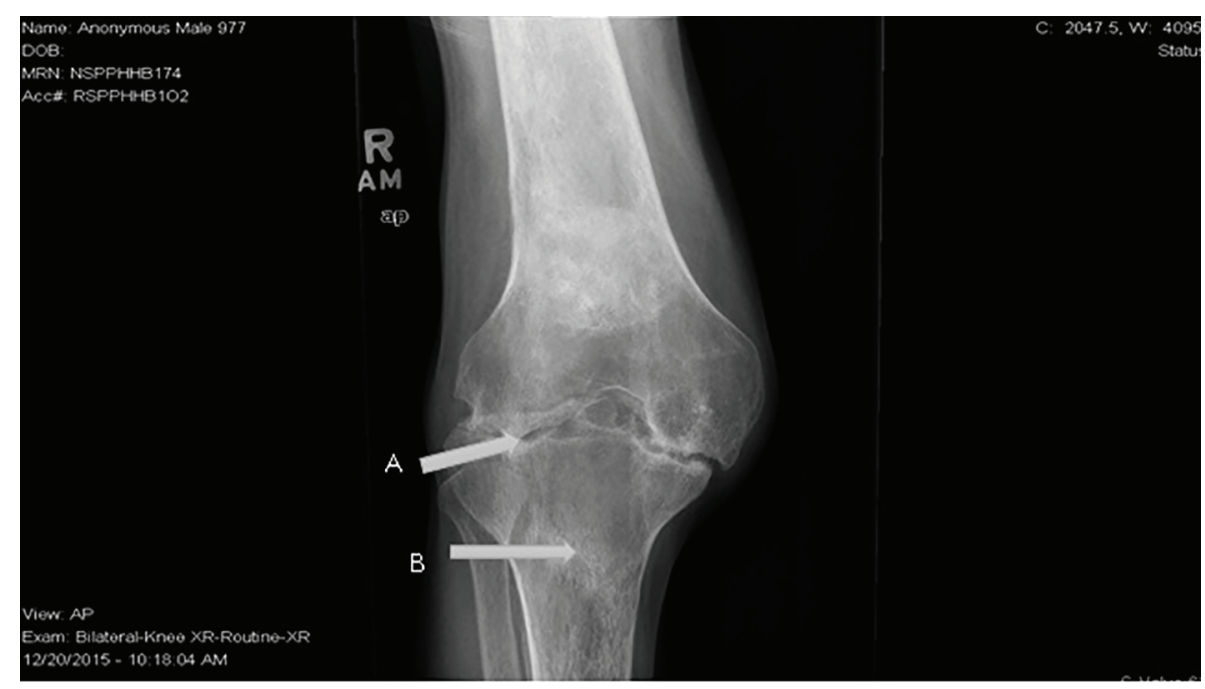

Figure 1. X-ray of right knee showing (A) erosive arthritis in the joint due to RA and (B) sclerosis in the tibial metaphysis due to SCD. 


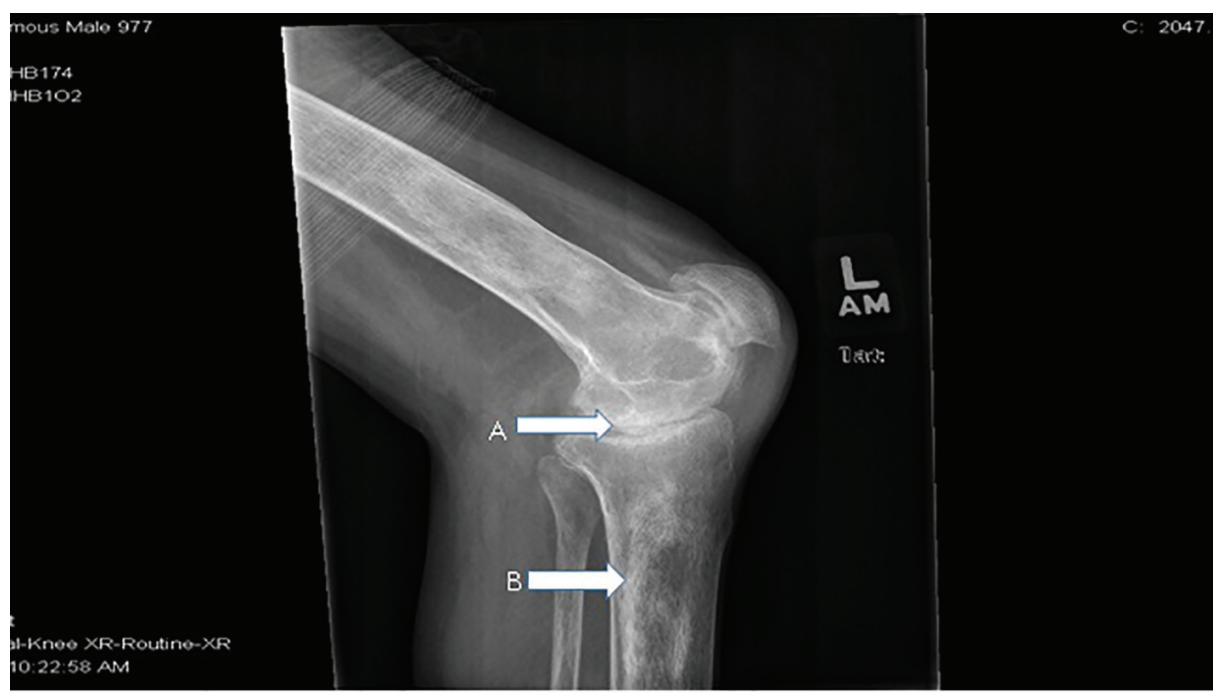

Figure 2. X-ray of left knee lateral view showing (A) erosive arthritis in the joint due to RA and (B) sclerosis in the diaphysis due to SCD.

not have significant musculoskeletal pain due to SCD prior to diagnosis of RA. She later developed increasing joint pain associated with digital deformities. Subsequent workup showed that she had concurrent RA. Hand X-ray shows erosive arthritis and subluxation at the metacarpophalangeal joints (Fig. 5). Her RA is being treated with prednisone, methotrexate and plaquenil.

\section{Case 3}

This patient developed RA at a young age. She had active disease with recurrent joint pain. She developed Boutonniere deformities in both hands and fixed flexion deformities of both elbows and wrists within the first few years of diagnosis. Xrays of both hands and toes showed erosive arthritis. Her RA was treated with prednisone, methotrexate and azathioprine.

\section{Case 4}

This patient had an added history of pulmonary embolism and chronic venous stasis ulcers. She had recurrent pain in the hips and knees which were attributed to SCD. Due to marked signs of inflammation in the knee, she underwent further diagnostic workup which revealed concurrent RA. Her RA is well controlled on sulfasalazine and plaquenil.

\section{Cases 5-8}

These patients have stable rheumatoid disease with less fre-

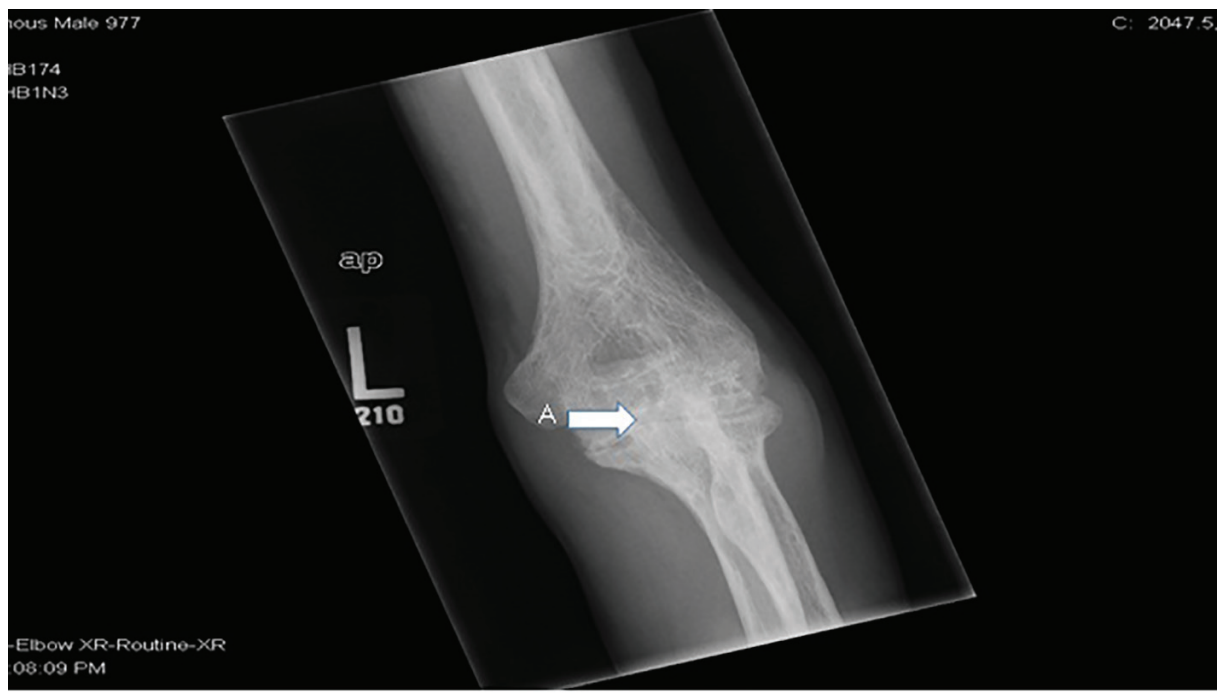

Figure 3. X-ray of left elbow showing (A) erosive RA. 


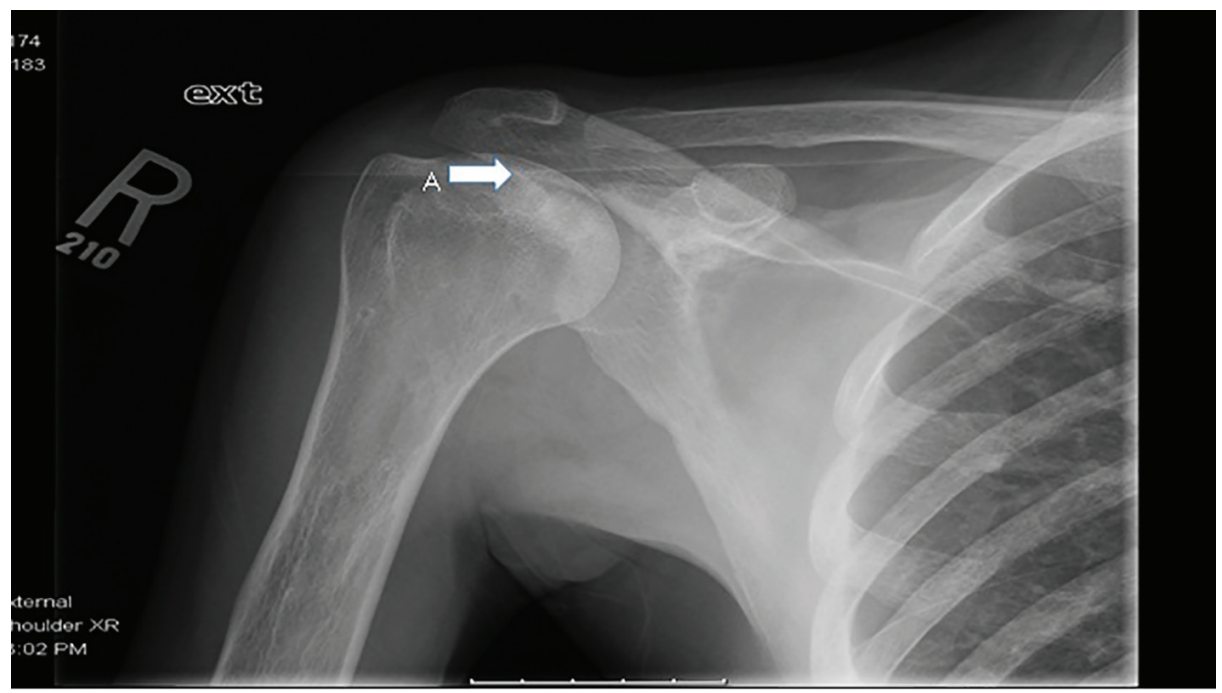

Figure 4. X-ray of right shoulder showing (A) avascular necrosis of humeral head with preserved articular surface.

quent joint pain. There were minimal articular changes on $\mathrm{X}$ ray. Cases 7 and 8 have been on leflunomide and 5 and 6 are not on any anti-rheumatic medications.

\section{Discussion}

We analyzed the number of ER visits and hospitalizations of these patients before and after diagnosis of RA. Patients with severe RA had a higher admission rate (Table 2) compared to patients with mild RA and both groups were treated as SCD painful crises. These painful events were retrospectively found not attributable to SCD based on clinical assessments, biomarkers and radiological findings. Both groups had similar hospitalization rates before diagnosis of RA. Case 3 had severe RA but relatively fewer admissions at our hospital after the di- agnosis of RA but she did report frequent overseas travel with hospitalizations in her home country. Case 6 had notably only a single hospitalization but she has a very recent diagnosis of RA.

In our case series of patients with SCD and concurrent RA, we elaborate the following differentiating features based on our observations.

\section{Clinical features}

In SCD, patients develop acute bone infarcts with localized pain, swelling, erythema and tenderness but articular involvement is not an initial finding. AVN is a bone infarct involving the epiphysis of a long bone, commonly the head of femur and humerus [8]. Acute episodes of AVN cause limitation of

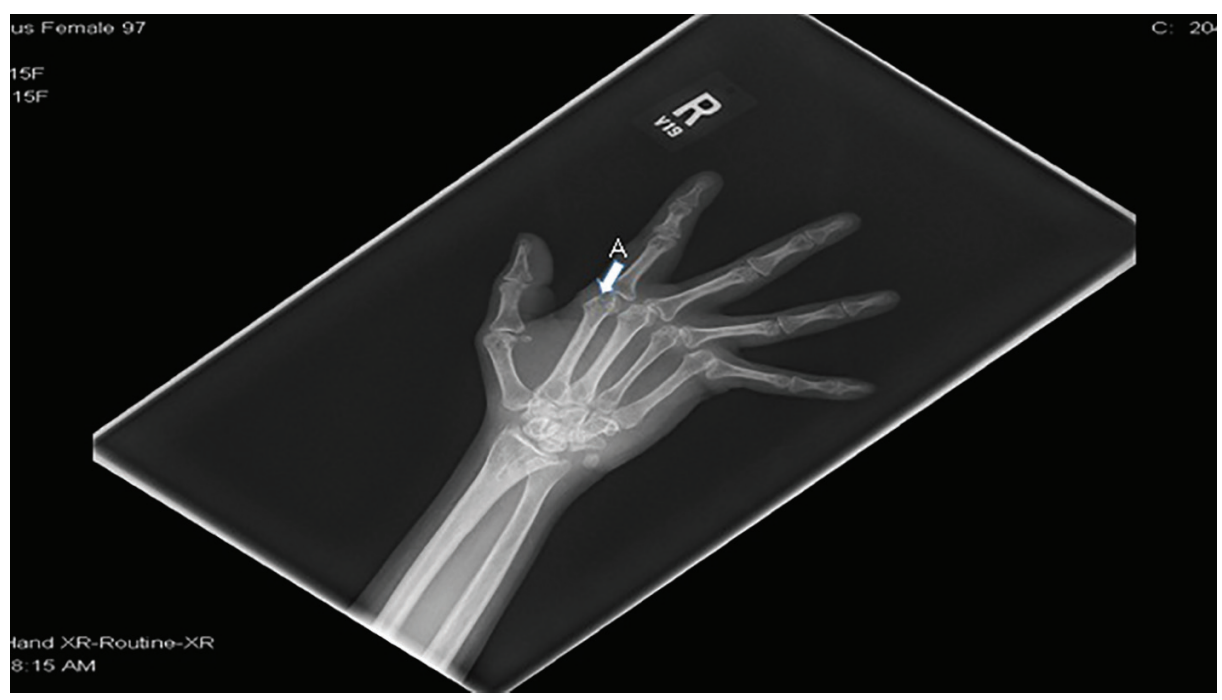

Figure 5. Right hand X-ray shows (A) erosive arthritis, subluxation at the metacarpophalangeal joints and periarticular osteopenia. 
Table 2. Number of Admissions for Painful Events Before and After Diagnosis of RA

\begin{tabular}{|c|c|c|c|c|c|c|c|c|}
\hline & Case 1 & Case 2 & Case 3 & Case 4 & Case 5 & Case 6 & Case 7 & Case 8 \\
\hline $\begin{array}{l}\text { Painful events requiring admission in the } 5 \text { years } \\
\text { period before diagnosis of RA }\end{array}$ & 2 & 1 & 0 & 0 & 0 & 9 & 1 & 0 \\
\hline $\begin{array}{l}\text { Painful events requiring admission in the } 5 \text { years } \\
\text { period after diagnosis of RA }\end{array}$ & 53 & 16 & 2 & 16 & 2 & 1 & 4 & 6 \\
\hline
\end{tabular}

Data only represent the available information from our institution.

joint movement due to pain but in the chronic phase, articular surface collapse can occur leading to secondary degenerative osteoarthrosis. In RA there is polyarticular, symmetrical joint inflammation with chronic pain, joint stiffness, joint line tenderness and synovial hypertrophy. Bone infarct in SCD involving phalanges is called dactylitis and is an acute process as compared to chronic small joint stiffness in RA. Dactylitis can occur in children but is not common in adults [9]. In RA, the small joints of the hands and feet are involved early in the course of disease and other synovial joints such as the elbows, shoulders, ankles and knees are usually affected later $[10,11]$. Periarticular involvement with tendon rupture is common in RA but is not seen in SCD. Vertebral central endplate depression causing H-shaped vertebral bodies is a classical sign of SCD but is never seen in RA. In making a distinction between these two conditions, particular attention must be placed on the temporal association of the joint symptoms, the pattern of joint involvement as mentioned above and the disease progression.

\section{Radiological signs}

Musculoskeletal symptoms in co-existent SCD and RA can pose a diagnostic challenge. In these instances, there are specific radiological features that can be used to distinguish underlying skeletal pathologies in each condition. These changes are well demonstrated in the X-rays of our patients (Figs. 1 and $2)$. In SCD, bone infarct due to vaso-occlusion is the primary pathology. X-rays initially show subchondral lucencies at the infarcted sites along with periosteal reaction. As the condition becomes more chronic, subchondral sclerosis develops. Repeated bone infarcts involving epiphyses lead to osteonecrosis (AVN) commonly of femoral and humeral heads [12, 13] which can eventually progress to depression, collapse, and fragmentation of the articular surface $[12,14]$. Overtime secondary degenerative changes of osteoarthrosis with eccentric joint space narrowing may occur. In RA, damage occurs at the articular surface. There is synovial inflammation with destruction of cartilage and formation of scar tissue at a later stage. This leads to concentric joint space narrowing consistent with inflammatory arthropathy. It can take several weeks to identify changes on conventional radiographs, as initial findings are very subtle [15]. In RA, activation of osteoclasts and alterations in the microcirculation cause para-articular osteoporosis as seen on X-rays. In advanced stages, there is joint subluxation with spine being the most commonly affected site [16]. MRI is more sensitive to detect changes early in both conditions. In SCD with acute bone infarcts, initial X-rays appear normal but MRI images (in particular, T2-weighted images) show regions of high signal intensity indicative of bone marrow edema [14]. AVN can also be picked up in early stages by MRI. In early RA, MRI shows erosions along with hypertrophic synovial tissue.

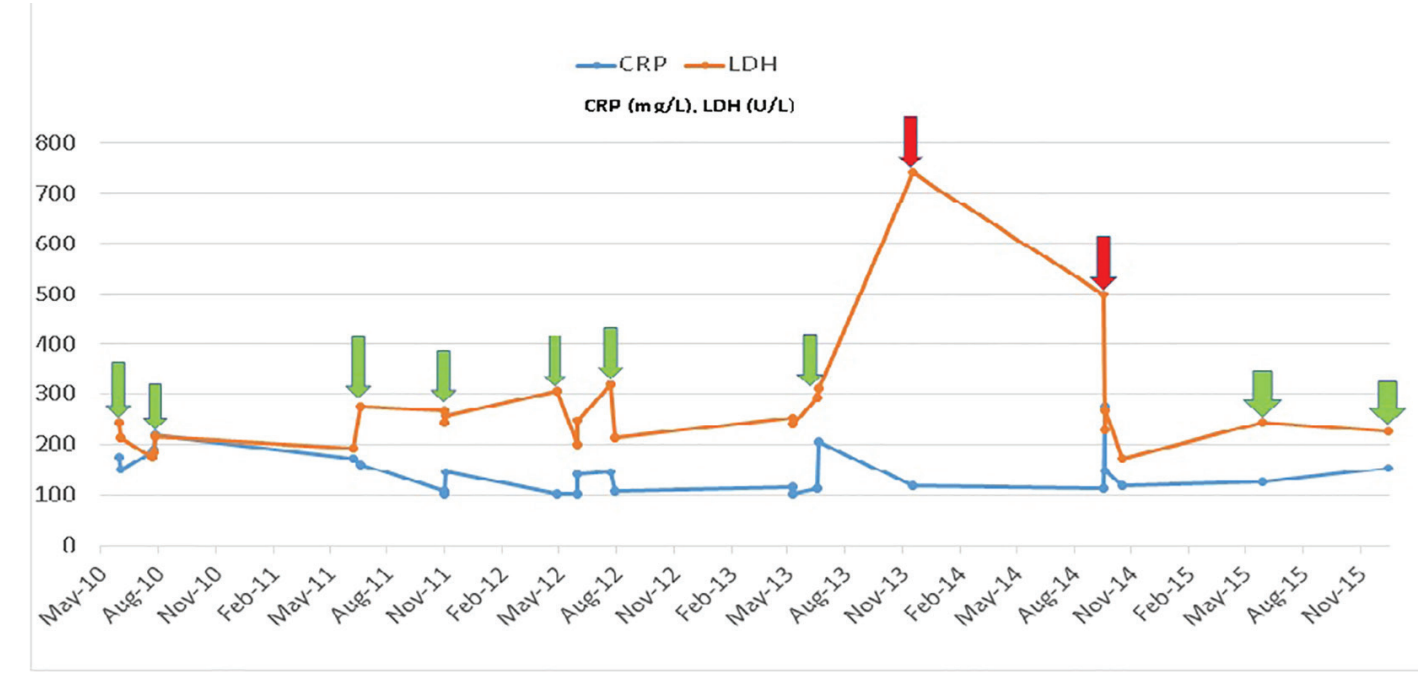

Figure 6. Comparison of biomarkers LDH and CRP in case 1 during admissions. Hospitalization for painful events due to sickle cell disease (red arrow). Hospitalization for painful events due to rheumatoid arthritis (green arrow). 


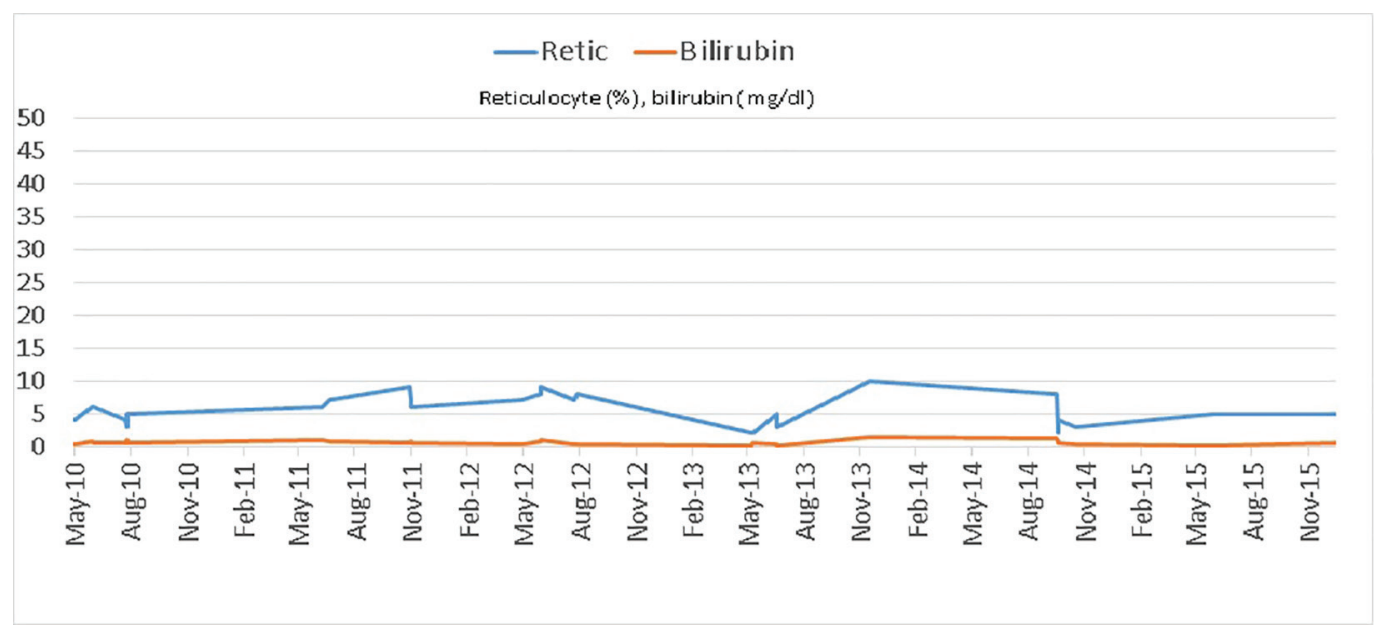

Figure 7. Reticulocyte count and total bilirubin levels in case 1 during admissions in a 5-year period.

\section{Biomarkers}

Serum markers are routinely used to assess disease activity in both conditions. Erythrocyte sedimentation rate (ESR) and C-reactive protein (CRP) are the most commonly used biomarkers. Elevated CRP is a non-specific indicator of both inflammation and necrosis in tissues. While CRP has its value in monitoring disease activity in each condition [17], it cannot reliably differentiate inflammation in SCD from that in RA. This can pose a challenge when patients with co-existent RA and SCD present with acute musculoskeletal symptoms. In our cohort of patients, we noted that an elevated CRP associated with a concurrent rise in lactate dehydrogenase (LDH), bilirubin and reticulocyte count above patient's baseline corresponds with active SCD. In active RA on the other hand, CRP elevation was associated with LDH, reticulocyte count and bilirubin levels remaining at baseline. This point is well demonstrated graphically for case 1 (Figs. 6 and 7). Also median CRP levels were higher in patients with severe RA as compared to patients with bone infarcts in SCD. A prospective cohort study in children showed that LDH levels increase significantly during vaso-occlusive crises compared with steady-state values and that there is a significant positive correlation between LDH levels and the severity of pain in SCD [18]. ESR is a rough measure of abnormal concentrations of acute phase proteins and immunoglobulins. This property makes ESR a sensitive, but non-specific, indicator of tissue damage and inflammation. ESR is used to assess RA severity. It is important to point out that the interpretation of ESR is of little relevance in SCD as sickled erythrocytes are unable to achieve Rouleau formation (underlying basis of test) which results in lower ESR values [19]. On the other hand, if the hematocrit is low, red cells can also aggregate and sediment faster giving rise to abnormally elevated ESR. Therefore, ESR cannot be used to assess RA disease activity in SCD.

\section{Treatment}

We make note of a few pertinent issues related to treatment in these patients. Hydroxyurea and methotrexate are both bone marrow suppressants. We are unable to find studies showing the safety of combined use of these two agents in patients suffering from these concurrent conditions. Therefore, our patients with SCD who benefited from hydroxyurea had to be switched to a less myelo-suppressive anti-rheumatic agent early in the course of RA treatment. Initially, a few of our patients noted that their sickle cell pain worsened after being started on methotrexate, the etiology of which is not clear. Careful evaluation of the painful events in patients with overlapping disease will enable an accurate diagnosis of RA exacerbations which when treated with appropriate anti-rheumatic agents will prevent disease progression and morbidity. Another notable point is that the use of steroids to treat RA in these patients may increase their risk of AVN as they have an inherent risk for it. There is also a noticeable high opiate usage in these patients for pain in the acute setting due to inaccurate attribution of the pain to SCD. This has the potential for consequent opioid dependence.

\section{Conclusion}

All painful events in SCD may not be related to it. RA and SCD can co-exist. There should be thoughtful analysis of painful events in these patients to prevent treatment delay and morbidity from RA.

\section{Acknowledgments}

This case series review was conducted at New York City Health and Hospitals/Kings County, Brooklyn, New York, USA.

\section{Conflicts of Interest}

The authors declare that there are no conflicts of interest in the publication of this paper. 


\section{References}

1. Bunn HF. Pathogenesis and treatment of sickle cell disease. N Engl J Med. 1997;337(11):762-769.

2. Hassell KL. Population estimates of sickle cell disease in the U.S. Am J Prev Med. 2010;38(4 Suppl):S512-521.

3. Ashley-Koch A, Yang Q, Olney RS. Sickle hemoglobin (HbS) allele and sickle cell disease: a HuGE review. Am J Epidemiol. 2000;151(9):839-845.

4. Brozovic M, Davies SC, Brownell AI. Acute admissions of patients with sickle cell disease who live in Britain. Br Med J (Clin Res Ed). 1987;294(6581):12061208.

5. Lee DM, Weinblatt ME. Rheumatoid arthritis. Lancet. 2001;358(9285):903-911.

6. Helmick CG, Felson DT, Lawrence RC, Gabriel S, Hirsch $\mathrm{R}$, Kwoh CK, Liang MH, et al. Estimates of the prevalence of arthritis and other rheumatic conditions in the United States. Part I. Arthritis Rheum. 2008;58(1):15-25.

7. Aletaha D, Neogi T, Silman AJ, Funovits J, Felson DT, Bingham CO, 3rd, Birnbaum NS, et al. 2010 rheumatoid arthritis classification criteria: an American College of Rheumatology/European League Against Rheumatism collaborative initiative. Ann Rheum Dis. 2010;69(9):1580-1588.

8. Neumayr LD, Aguilar C, Earles AN, Jergesen HE, Haberkern CM, Kammen BF, Nancarrow PA, et al. Physical therapy alone compared with core decompression and physical therapy for femoral head osteonecrosis in sickle cell disease. Results of a multicenter study at a mean of three years after treatment. J Bone Joint Surg Am. 2006;88(12):2573-2582.

9. Watson RJ, Burko H, Megas H, Robinson M. The hand- foot syndrome in sickle-cell disease in young children. Pediatrics. 1963;31:975-982.

10. Fleming A, Crown JM, Corbett M. Early rheumatoid disease. I. Onset. Ann Rheum Dis. 1976;35(4):357-360.

11. Jacoby RK, Jayson MI, Cosh JA. Onset, early stages, and prognosis of rheumatoid arthritis: a clinical study of 100 patients with 11-year follow-up. $\mathrm{Br}$ Med J. 1973;2(5858):96-100.

12. Ganguly A, Boswell W, Aniq H. Musculoskeletal manifestations of sickle cell anaemia: a pictorial review. Anemia. 2011;2011:794283.

13. Lonergan GJ, Cline DB, Abbondanzo SL. Sickle cell anemia. Radiographics. 2001;21(4):971-994.

14. Ejindu VC, Hine AL, Mashayekhi M, Shorvon PJ, Misra RR. Musculoskeletal manifestations of sickle cell disease. Radiographics. 2007;27(4):1005-1021.

15. Sommer OJ, Kladosek A, Weiler V, Czembirek H, Boeck M, Stiskal M. Rheumatoid arthritis: a practical guide to state-of-the-art imaging, image interpretation, and clinical implications. Radiographics. 2005;25(2):381-398.

16. Resnick D, Kyriakos M, Greenway GD. Rheumatoid arthritis. Diagnosis of Bone and joint disorders. 2002; 2: 891-974.

17. Niu X, Chen G. Clinical biomarkers and pathogenic-related cytokines in rheumatoid arthritis. J Immunol Res. 2014;2014:698192.

18. Neely CL, Wajima T, Kraus AP, Diggs LW, Barreras L. Lactic acid dehydrogenase activity and plasma hemoglobin elevations in sickle cell disease. Am J Clin Pathol. 1969;52(2):167-169.

19. Damanhouri GA, Jarullah J, Marouf S, Hindawi SI, Mushtaq G, Kamal MA. Clinical biomarkers in sickle cell disease. Saudi J Biol Sci. 2015;22(1):24-31. 